\title{
Natural autoxidation of longifolene and anti-termite activities of the products
}

\author{
Akisato Mukai $^{1} \cdot$ Koetsu Takahashi $^{1,2} \cdot$ Tatsuya Ashitani $^{1,2}$ (])
}

Received: 2 February 2017/Accepted: 8 May 2017/Published online: 1 June 2017

(C) The Japan Wood Research Society 2017

\begin{abstract}
Here, we investigated the reaction mechanism of longifolene autoxidation and the bioactivities of its products. A longifolene sample was autoxidized at $120^{\circ} \mathrm{C}$ or room temperature, and the products were analyzed by gas chromatography. Longicamphenylone, norlongilactone, isolongifolic acid, longifolic acid, and longifolene-hydroxy ketone were detected as the products in the autoxidation at $120{ }^{\circ} \mathrm{C}$. During the reaction, longifolene was converted into the other autoxidation products via the formation of longifolene oxide as an intermediate. Anti-termite activities of the longifolene and its autoxidation products were examined against a Japanese subterranean termite (Reticulitermes speratus Kolbe) by paper disk methods in Petri dishes. Longifolene and the autoxidation products exhibited anti- $R$. speratus activities, and all the autoxidation products had stronger termiticidal and antifeedant activities than longifolene.
\end{abstract}

Keywords Bioactivity $\cdot$ Longifolene $\cdot$ Natural autoxidation $\cdot$ Sesquiterpene $\cdot$ Termite

Part of this report was presented at the 65th Annual Meeting of the Japan Wood Research Society in Tokyo, March 2015, and the International Chemical Congress of Pacific Basin Societies 2015, Honolulu, December 2015.

Tatsuya Ashitani

ashitani@tds1.tr.yamagata-u.ac.jp

Akisato Mukai

akisato.mukai@gmail.com

1 The United Graduate School of Agricultural Science, Iwate University, Morioka 020-8550, Japan

2 Faculty of Agriculture, Yamagata University, 1-23 Wakabamachi, Tsuruoka, Yamagata 997-8555, Japan

\section{Introduction}

Many components in plant extracts have various functions, including anti-fungal, anti-insect, and insect-attractant functions [1]. However, the functions of some compounds have not been studied in detail, even though they exist in large quantities in plants. Especially, sesquiterpene hydrocarbons are well-known plants components, but studies of their bioactivities are limited. In our previous report, the sesquiterpene hydrocarbons, with high steric hindrances, of caryophyllene and humulene were autoxidized easily by sulfur or oxygen $[2,3]$. The oxidized products had anti-termite and anti-tick bioactivities [4, 5]. Other sesquiterpene hydrocarbons with high steric hindrance are also autoxidized easily. For example, longifolene and thujopsene-a tricyclic sesquiterpene-are autoxidized following exposure to air [6-8]. It is one of the characteristic functions of these sesquiterpene hydrocarbons. The oxidation occurred in natural condition is an easy synthesis method to convert into useful bioactive compounds. In addition, the natural autoxidation has the advantage that the reaction proceeds automatically without catalyst and special instrument [7], as compared with other methods such as hydroxylation, isomerization and so on. Therefore, we have investigated the autoxidation to enhance utility of the sesquiterpene hydrocarbons.

Longifolene is a main sesquiterpene component of Pinus densiflora and Pinus thunbergii, which are widespread in Japan $[9,10]$. For example, essential oil of $P$. thunbergii heart wood contained $30.5 \%$ of longifolene and component in sesquiterpene, part of the essential oil was almost longifolene in our previous study [11]. Low-polarity extracts of pine wood consist mainly of terpenoid components, and they are useful products. Monoterpenes, such as $\alpha$-pinene and $\beta$-pinene, in the extracts are used as flavor 
and/or medical materials, and the resin components of diterpenes are used as sizing reagents during pulping [12]. However, longifolene, which accounts for most of the sesquiterpenes in the extracts, has not been regarded as a useful component compared with the mono- and di-terpenes. Thus, characterization of longifolene will not only lead to the elucidation of the terpenoid reaction, but it will also enhance the use of Pinus wood extracts. Longifolene has been reported to undergo oxidation by reacting with lead tetraacetate [13], via ozonolysis in a chloroform solution [14], and by reacting with perbenzoic acid [15] and permanganate [16], which results in oxidation products such as longicamphenylone. Natural autoxidation is an ecologically safe and attractive method for converting longifolene to useful substances because the reaction can be carried out without harmful catalysts and solvents. However, the mechanism for the natural autoxidation of longifolene, as well as the bioactivities of its products, has not been reported in detail. Although longifolene autoxidation has only been examined at room temperature, it is possible that the reaction can be accelerated or controlled by heating. About bioactivity of autoxidation products, we reported that oxidized products had higher termiticidal, anti-feeding, and repellent activities than material in a case of caryophyllene against Reticulitermes speratus Kolbe [4]. $R$. speratus is one of the representative termite species for wood material in Japan. $P$. densiflora and thunbergii woods are comparatively weaker than other conifer woods such as Cryptomeria japonica and Chamaecyparis obtusa against the termite. If longifolene contained in the pine woods can be easily converted into anti-termitic substances by autoxidation, it will be important knowledge for development of new methods to improve durability of the pine woods by effective utilization of the extractive components. Therefore, to obtain the fundamental knowledge for longifolene autoxidation, we examined the reaction at high temperatures and the bioactivities of its products against termites.

\section{Materials and methods}

\section{Isolation of longifolene}

A pine oil sample obtained from the root of Pinus thunbergii was supplied by Dr. Nagahama, Professor Emeritus at Sojo University. The sample was subjected to Kugelrohr distillation. The distillate $\left(130-150{ }^{\circ} \mathrm{C}, 0.2 \mathrm{~mm} \mathrm{Hg}\right)$ was separated by silica gel column chromatography with hexane as the solvent to obtain longifolene [98\% purity, as assessed by gas chromatography (GC)].

\section{Analysis of compounds}

A GC-flame ionization detector (GC-FID) analysis was performed with a Shimadzu GC-2014 gas chromatograph under the following conditions: a DB-1 capillary column $(30 \mathrm{~m} \times 0.32 \mathrm{~mm}$ inside diameter; $0.25 \mu \mathrm{m}$ film thickness; J\&W Scientific, Folsom, CA, USA); a column temperature ranging from 100 to $200{ }^{\circ} \mathrm{C}(0 \mathrm{~min})$ at $5{ }^{\circ} \mathrm{C} / \mathrm{min}$ and from 200 to $320{ }^{\circ} \mathrm{C}(2 \mathrm{~min})$ at $10{ }^{\circ} \mathrm{C} / \mathrm{min}$; an injection temperature of $230{ }^{\circ} \mathrm{C}$; and a detection temperature of $320^{\circ} \mathrm{C}$. GC-mass spectrometry (GC-MS) data were collected with a Shimadzu QP-2010 Ultra gas chromatographmass spectrometer under the following conditions: a DB-1 capillary column $(0.32 \mathrm{~mm}$ i.d. $\times 30 \mathrm{~m} ; 0.25-\mu \mathrm{m}$ film thickness; J\&W Scientific); a column temperature ranging from $100{ }^{\circ} \mathrm{C}(1 \mathrm{~min})$ to $200{ }^{\circ} \mathrm{C}(0 \mathrm{~min})$ at $5{ }^{\circ} \mathrm{C} / \mathrm{min}$ and from $200{ }^{\circ} \mathrm{C}(0 \mathrm{~min})$ to $320^{\circ} \mathrm{C}(2 \mathrm{~min})$ at $10{ }^{\circ} \mathrm{C} / \mathrm{min}$; an injection temperature of $250{ }^{\circ} \mathrm{C}$; an interface temperature of $300{ }^{\circ} \mathrm{C}$; and an acquisition mass range of 50-450 atomic mass units using helium as the carrier gas. To analyze carboxylic compounds, a sample was injected into the gas chromatograph after methylation by trimethylsilyldiazomethane (GL Science). Nuclear magnetic resonance (NMR) spectra were measured with a JEOL JNM-EX400 $\left({ }^{1} \mathrm{H} 400 \mathrm{MHz} /{ }^{13} \mathrm{C} 100 \mathrm{MHz}\right)$ spectrometer. Longicamphenylone, norlongilactone, and longicamphenylol were identified by comparing the experimental GC-MS data with authentic compounds obtained in our previous study [7].

\section{Autoxidation}

Longifolene was stirred in a flask attached to a condenser while exposed to air at 100,120 , or $140{ }^{\circ} \mathrm{C}$ in a glycerin bath. A portion of the reaction mixture was collected with a capillary at each reaction time $(1,2,4,8,12$, and $24 \mathrm{~h})$. In the non-heating method, longifolene was placed in the dark at room temperature for 3 years. Each reaction product was analyzed by GC-MS and GC-FID. Heneicosane and veratric acid were used as internal standard in GC analysis for neutral and acidic compounds, respectively. The yields of each product and unreacted longifolene were calculated by calibration curves prepared from GC-FID analysis of the internal standard and each compound.

In the heating method, the reaction sample at $120^{\circ} \mathrm{C}$ after $24 \mathrm{~h}$ was dissolved in ethyl acetate, and the solution was washed with a $1 \% \mathrm{NaOH}$ solution. The neutral fraction obtained after evaporation of the organic layer was applied to a silica gel column with hexane and ethyl acetate to isolate longicamphenylone and norlongilactone. The acidic fraction obtained after acidification of the alkaline wash- 
Fig. 1 Structures of longifolene and its autoxidation products. $\mathbf{L}$, longifolene; LC,

longicamphenylone; LL, norlongilactone; LA, longicamphenylol; LAc, longifolic acid; isoLAc, isolongifolic acid; LOX, longifolene oxide; LHK, longifolene-hydroxy ketone

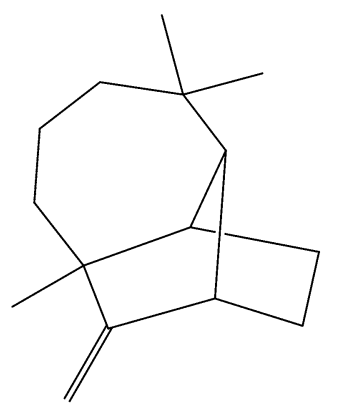

$\mathbf{L}$

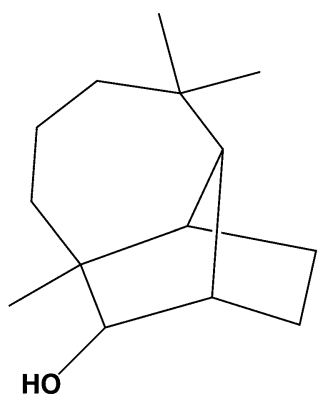

LA

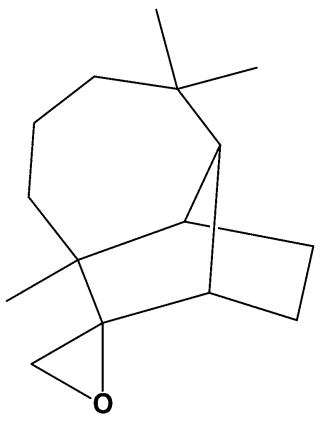

LOX

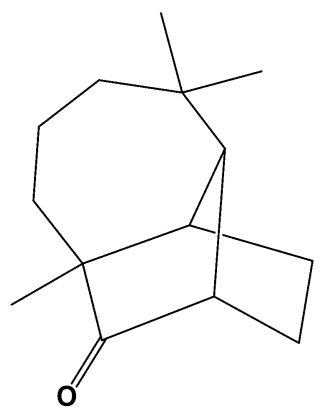

LC

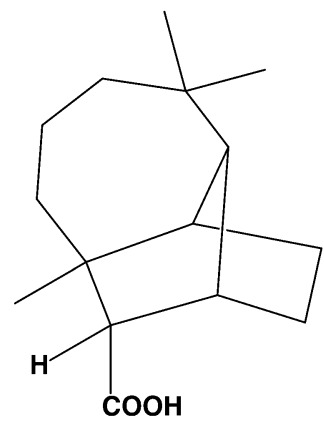

LAc

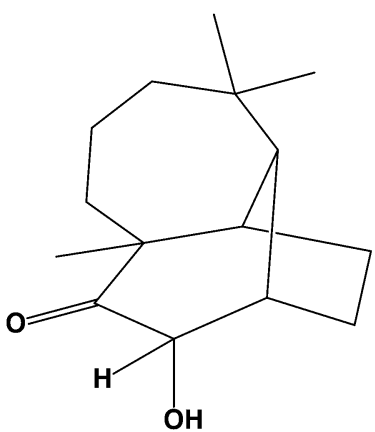

LHK

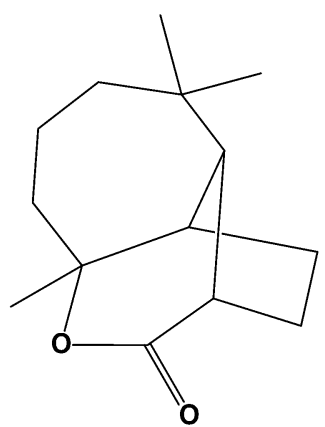

LL

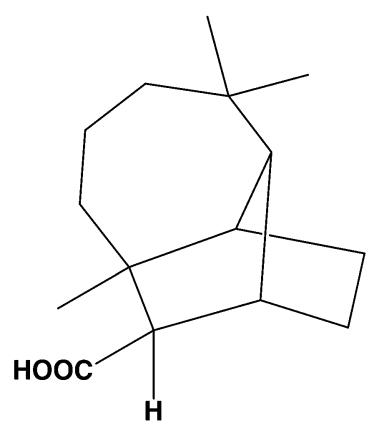

isoLAc ings was applied to a silica gel column with hexane:diethyl ether (50:4) to isolate longifolic acid and isolongifolic acid. Longifolic acid and isolongifolic acid were identified by comparing their ${ }^{1} \mathrm{H}$ or ${ }^{13} \mathrm{C}$ NMR spectra with reference data [14].

\section{Oxidation of longifolene with $\boldsymbol{m}$-chloroperbenzoic acid}

Longifolene was reacted with $m$-chloroperbenzoic acid in benzene or chloroform at room temperature for $24 \mathrm{~h}$ according to references $[17,18]$. The reaction mixture was washed with $1 \% \mathrm{NaOH}$. The solvent was removed under low pressure by a rotary evaporator. The longifolene oxide was obtained from the reaction in benzene, and identified by comparing its ${ }^{1} \mathrm{H}$ or ${ }^{13} \mathrm{C}$ NMR spectra with reference data. During the reaction in chloroform, longifolene-hydroxy ketone was formed, and it was purified by recrystallization with ethanol. Structural confirmation of the longifolene-hydroxy ketone was obtained by an NMR analysis.

\section{Longifolene-hydroxy ketone}

${ }^{1} \mathrm{H}-\mathrm{NMR}\left(400 \mathrm{MHz}, \mathrm{CDCl}_{3}\right): \delta 1.09(6 \mathrm{H}, \mathrm{s}), 1.15(3 \mathrm{H}, \mathrm{s})$, 1.30-1.40 (2H, m), 1.41-1.85 (8H, m), 2.23-2.30 (1H, m), $2.52-2.60(1 \mathrm{H}, \mathrm{m}), 3.68(1 \mathrm{H}, \mathrm{d}, J=2.5 \mathrm{~Hz}), 4.58-4.65$ $(1 \mathrm{H}, \mathrm{m}) ;{ }^{13} \mathrm{C}-\mathrm{NMR}\left(100 \mathrm{MHz}, \mathrm{CDCl}_{3}\right): 21.5\left(\mathrm{CH}_{2}\right), 21.9$ 
Fig. $2 \mathrm{HMBC}$ and NOESY correlations observed in the NMR analysis of longifolenehydroxy ketone acetate

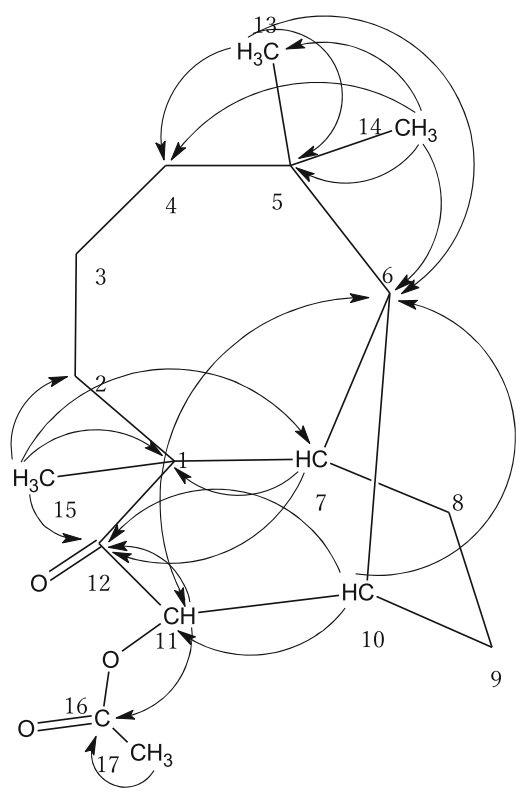

HMBC

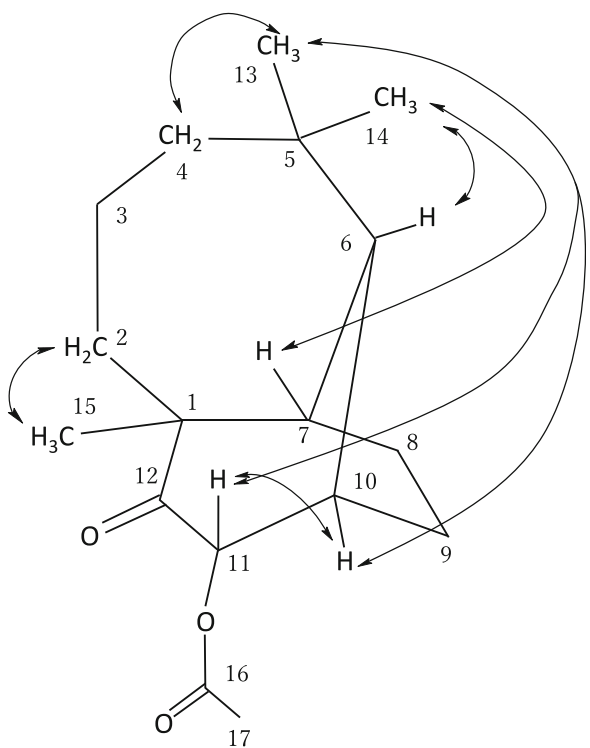

NOESY
Table 1 Yield* of autoxidation products and unreacted longifolene from reactions at $120{ }^{\circ} \mathrm{C}$ and room temperature

\begin{tabular}{|c|c|c|c|c|c|c|c|c|}
\hline \multicolumn{2}{|l|}{ Conditions } & \multicolumn{6}{|c|}{ Products** $(\%)$} & \multirow{2}{*}{$\begin{array}{l}\text { Unreacted material }^{* *}(\%) \\
\mathbf{L}\end{array}$} \\
\hline Temperature & Time & LC & LA & $\mathbf{L L}$ & LAc & isoLAc & LHK & \\
\hline $120^{\circ} \mathrm{C}$ & $24 \mathrm{~h}$ & 23 & - & 4.3 & 3.0 & 1.3 & 2.2 & 22 \\
\hline Room temperature & 3 years & 23 & 2.1 & 3.0 & 6.2 & 1.4 & - & 46 \\
\hline
\end{tabular}

* Each yield was calculated by dividing the molar amount of product or unreacted longifolene by the molar amount of longifolene before reaction

** L, longifolene; LC, longicamphenylone; LL, norlongilactone; LA, longicamphenylol; LAc, longifolic acid; isoLAc, isolongifolic acid; LHK, longifolene-hydroxy ketone; 100\% = material longifolene
$\left(\mathrm{CH}_{2}\right), 26.4\left(\mathrm{CH}_{3}\right), 27.5\left(\mathrm{CH}_{2}\right), 29.6\left(\mathrm{CH}_{3}\right), 33.7\left(\mathrm{CH}_{3}\right)$, $34.1(\mathrm{C}), 41.4\left(\mathrm{CH}_{2}\right), 42.0\left(\mathrm{CH}_{2}\right), 43.3(\mathrm{CH}), 45.1(\mathrm{CH})$, $50.7(\mathrm{C}), 56.5(\mathrm{CH}), 73.9(\mathrm{CH}), 217.2(\mathrm{C})$.

The longifolene-hydroxy ketone was acetylated by acetic anhydride in pyridine to analyze its configuration. The longifolene-hydroxy ketone acetate product was analyzed by obtaining ${ }^{1} \mathrm{H}-\mathrm{NMR},{ }^{13} \mathrm{C}-\mathrm{NMR}$, heteronuclear multiple quantum correlation (HMQC), heteronuclear multiple bond correlation (HMBC), and nuclear overhauser effect spectroscopy (NOESY) spectra.

\section{Longifolene-hydroxy ketone acetate}

${ }^{1} \mathrm{H}-\mathrm{NMR}\left(400 \mathrm{MHz}, \mathrm{CDCl}_{3}\right): \delta 1.07(3 \mathrm{H}, \mathrm{s}), 1.10(3 \mathrm{H}, \mathrm{s})$, $1.15(3 \mathrm{H}, \mathrm{s}), 1.48-1.81(11 \mathrm{H}, \mathrm{m}), 2.13(3 \mathrm{H}, \mathrm{s}), 2.25(1 \mathrm{H}$, bs), $2.48-2.50(1 \mathrm{H}, \mathrm{m}), 5.69(1 \mathrm{H}, \mathrm{dd}, J=3.7,1.3 \mathrm{~Hz})$; ${ }^{13} \mathrm{C}$-NMR $\left(100 \mathrm{MHz}, \mathrm{CDCl}_{3}\right): 20.9\left(\mathrm{CH}_{3}\right), 21.3\left(\mathrm{CH}_{2}\right)$, $23.0\left(\mathrm{CH}_{2}\right), 26.7\left(\mathrm{CH}_{3}\right), 27.5\left(\mathrm{CH}_{2}\right), 29.8\left(\mathrm{CH}_{2}\right), 33.4$ $\left(\mathrm{CH}_{3}\right), 34.2(\mathrm{C}), 41.1(\mathrm{CH}), 41.4\left(\mathrm{CH}_{2}\right), 41.7\left(\mathrm{CH}_{2}\right), 44.3$ $(\mathrm{CH}), 51.7(\mathrm{C}), 56.7(\mathrm{CH}), 75.9(\mathrm{CH}), 170.4(\mathrm{C}), 210.8(\mathrm{C})$.

\section{Termites}

Colonies of $R$. speratus Kolbe were collected from the wild in Tsuruoka, Yamagata, Japan, in 2010, and they were maintained in humidified chambers at $26 \pm 1{ }^{\circ} \mathrm{C}$.

\section{Anti-termite test}

We used no-choice and dual-choice methods for the antitermite tests, as described previously $[4,19,20]$. The nochoice test was employed to evaluate the termiticidal and antifeedant activity. Test samples were dissolved in acetone and applied to paper disks (8-mm diameter, $1.5-\mathrm{mm}$ thickness) at certain concentrations (sample weight/paper disk weight). The dual-choice test was used to investigate termiticidal and repellent activity. The dual-choice test was performed according to previous studies [4, 18]. A control disk was placed together with a sample-impregnated paper disk on sea sand in a Petri dish. Control disks were dosed only with pure acetone. In control test, a control disk was 
Table 2 Time course of the yield* of products during longifolene autoxidation at $120{ }^{\circ} \mathrm{C}$

\begin{tabular}{lllllll}
\hline Time (h) & \multicolumn{3}{l}{ Products** $(\%)$} & \multicolumn{2}{l}{ Unreacted material** (\%) } \\
\cline { 2 - 6 } & LC & LL & LAc & isoLAc & LHK & L \\
\hline 1 & 0.50 & 0.58 & - & - & - & $<99$ \\
2 & 0.93 & 0.53 & - & - & - & $<99$ \\
4 & 2.4 & 0.86 & - & - & - & 87 \\
8 & 7.5 & 1.7 & 1.1 & - & 0.79 & 71 \\
12 & 17 & 3.4 & 2.3 & 1.1 & 1.6 & 62 \\
24 & 23 & 4.3 & 3.0 & 1.3 & 2.2 & 22 \\
\hline
\end{tabular}

* Each yield was calculated by dividing the molar amount of product or unreacted longifolene by the molar amount of longifolene before reaction

** L, longifolene; LC, longicamphenylone; LL, norlongilactone; LAc, longifolic acid; isoLAc, isolongifolic acid; LHK, longifolene-hydroxy ketone

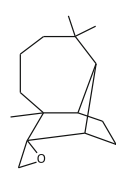

LOX
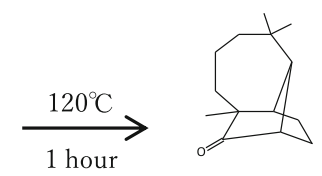

LC

$(4.5 \%)$

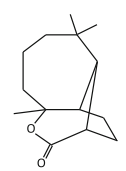

LL

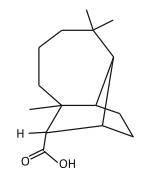

LAc

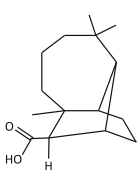

isoLAc

$(1.7 \%)$

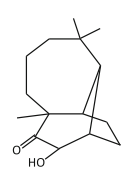

LHK

$(1.2 \%)$
Fig. 3 Autoxidation products of longifolene oxide at $120{ }^{\circ} \mathrm{C}$ and their yields. Each yield was calculated by dividing the molar amount by the molar amount of longifolene oxide before reaction. LC,

placed in each Petri dish. In the no-feed test, no paper disks were placed in the Petri dishes. Ten $R$. speratus workers were placed in each Petri dish, and the dishes were kept in a dark room for 21 days at $26 \pm 1{ }^{\circ} \mathrm{C}$. Three replicates of each treatment were prepared. Dead termites were removed daily, and the percent mortality rate was calculated. After 21 days, the sample disks were vacuum dried and weighed, and feeding activity was estimated by calculating the mass loss (percent weight loss) by:

Termite mortality $(\%)=100 \times($ Number of dead termites $/$ number of initial termites),

Mass loss $(\%)=100 \times($ Pre-treatment paper disk weight

- post-treatment paper disk weight)/

pre-treatment paper disk weight.

\section{Statistical analysis}

Mortality and weight loss for each treatment were calculated and compared by analysis of variance and a protected Tukey-Kramer test using Statcel 2 software (OMS Inc., Japan). Weight losses in each treatment were compared using a $t$ test, and $p<0.05$ was considered significant. longicamphenylone; LL, norlongilactone; LAc, longifolic acid; isoLAc, isolongifolic acid; LOX, longifolene oxide; LHK, longfolene -hydroxy ketone

\section{Results and discussion}

\section{Autoxidation of longifolene}

We examined longifolene autoxidation by heating and nonheating (room temperature) methods. Figure 1 shows the structures of longifolene ( $\mathbf{L}$ ) and the identified autoxidation products in this study. To accelerate and control the autoxidation, $\mathbf{L}$ was heated in the presence of air, without solvent and catalysis. In the heating method, the reaction temperature was examined from 100 to $140{ }^{\circ} \mathrm{C}$. For the reaction at $140{ }^{\circ} \mathrm{C}$, the rotation of the stirring bar was stopped by the solidification of $\mathbf{L}$ in the early stage, and the reaction did not proceed. The reaction at $120{ }^{\circ} \mathrm{C}$ showed higher product yields than the reaction at $100{ }^{\circ} \mathrm{C}$, as determined by GC. Thus, the reaction at $120{ }^{\circ} \mathrm{C}$ was further analyzed. After $24 \mathrm{~h}$, the amount of $\mathbf{L}$ in the reaction mixture decreased, and longicamphenylone (LC), norlongilactone (LL), longifolic acid (LAc), isolongifolic acid (isoLAc), and longifolene-hydroxy ketone (LHK) were produced. LHK is a well-known product of several $\mathbf{L}$ oxidation reactions, but its configuration is unclear [15, 21-23]. We examined the stereo-structure of LHK by an NMR analysis of its acetate derivative. Figure 2 shows the HMBC and NOESY spectra of longifolene-hydroxy ketone acetate. NOESY signal was observed between 


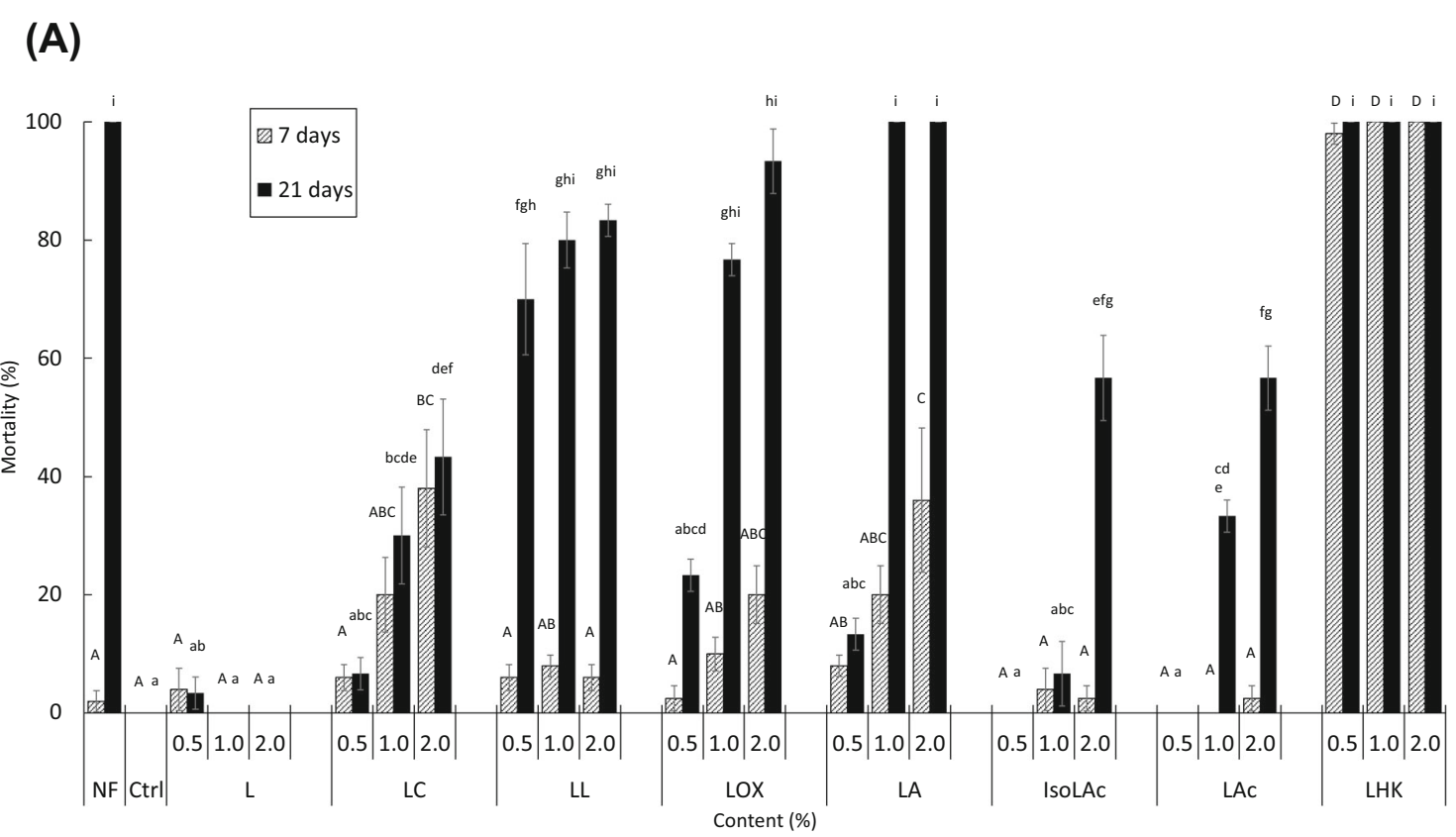

(B)

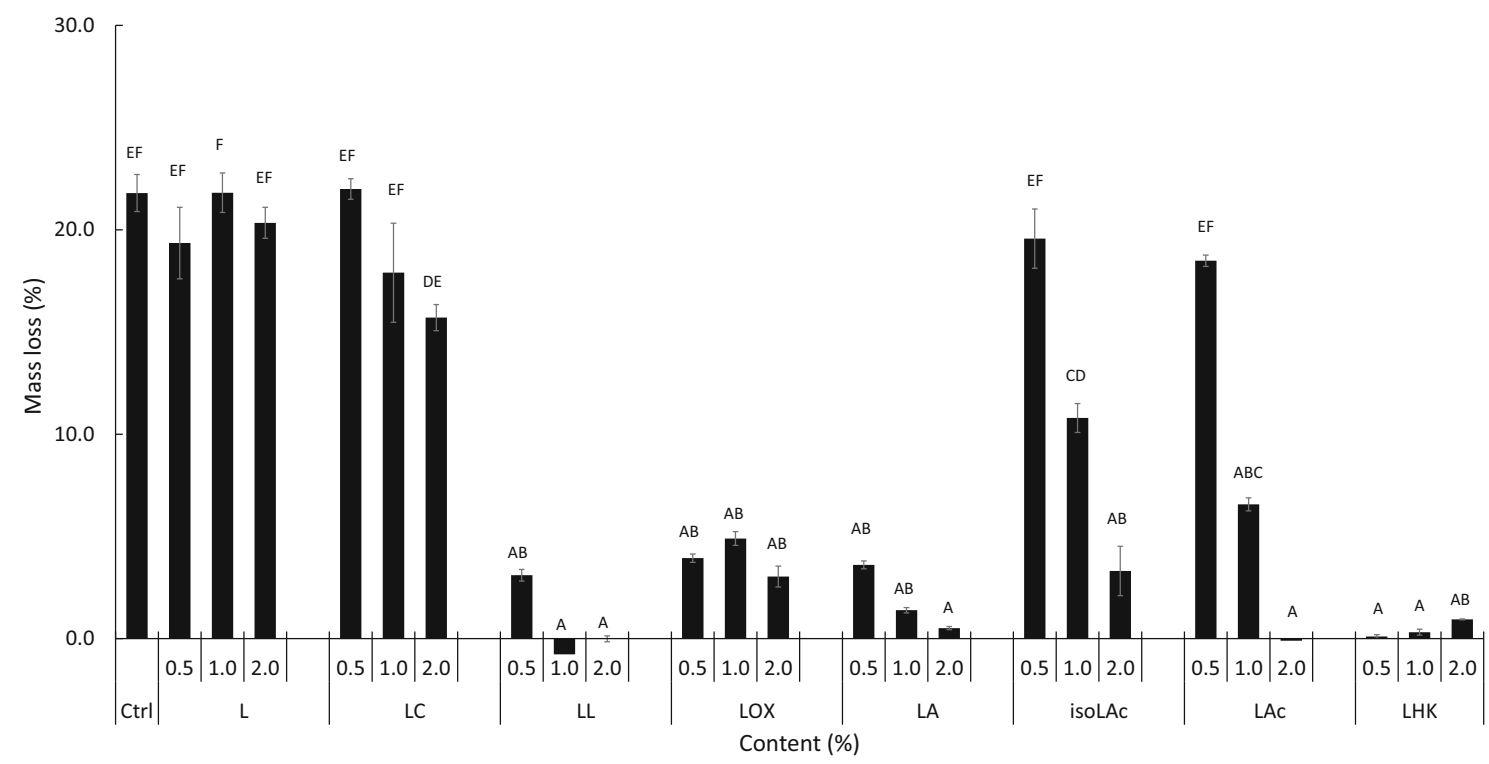

Fig. 4 a Termiticidal activities after 7 and 21 days and $\mathbf{b}$ antifeedant activities after 21 days of exposure to compounds in the no-choice test. Ctrl, control test; $\mathbf{L}$, longifolene; $\mathbf{L C}$, longicamphenylone; $\mathbf{L L}$, norlongilactone; LA, longicamphenylol; LAc, longifolic acid; LOX, longifolene oxide; isoLAc, isolongifolic acid; LHK, longifolene-

hydrogens at 11-C and 13-C in Fig. 2. The configuration of the 11-C bound acetate group was designated as $S$ according to the results of the NOESY spectra in Fig. 2 and the structure of LHK in Fig. 1.

In the non-heating method, when $\mathbf{L}$ was placed at room temperature for 3 years, $\mathbf{L}, \mathbf{L C}, \mathbf{L L}, \mathbf{L A c}$, isoLAc and $\mathbf{L A}$ were detected as the main products, and $\mathbf{L C}$ had the highest yield. Table 1 shows the yields of the main hydroxy ketone; NF, no-feed test. Bars indicate standard errors. Common letters indicate that there is no significant difference in the values. Different letters indicate statistically significant differences; Tukey-Kramer test, $p \leq 0.05$

products of $\mathbf{L}$ autoxidation by the heating and room temperature reactions. LA was not detected following autoxidation at $120^{\circ} \mathrm{C}$. It was considered that the $\mathbf{L A}$ was converted into $\mathbf{L C}$ by further oxidation under high temperature condition. In contrast, LHK was not detected following autoxidation at room temperature. As formation of LHK, rearrangement with ring expansion occurred in addition to oxidation. Therefore, high temperature 


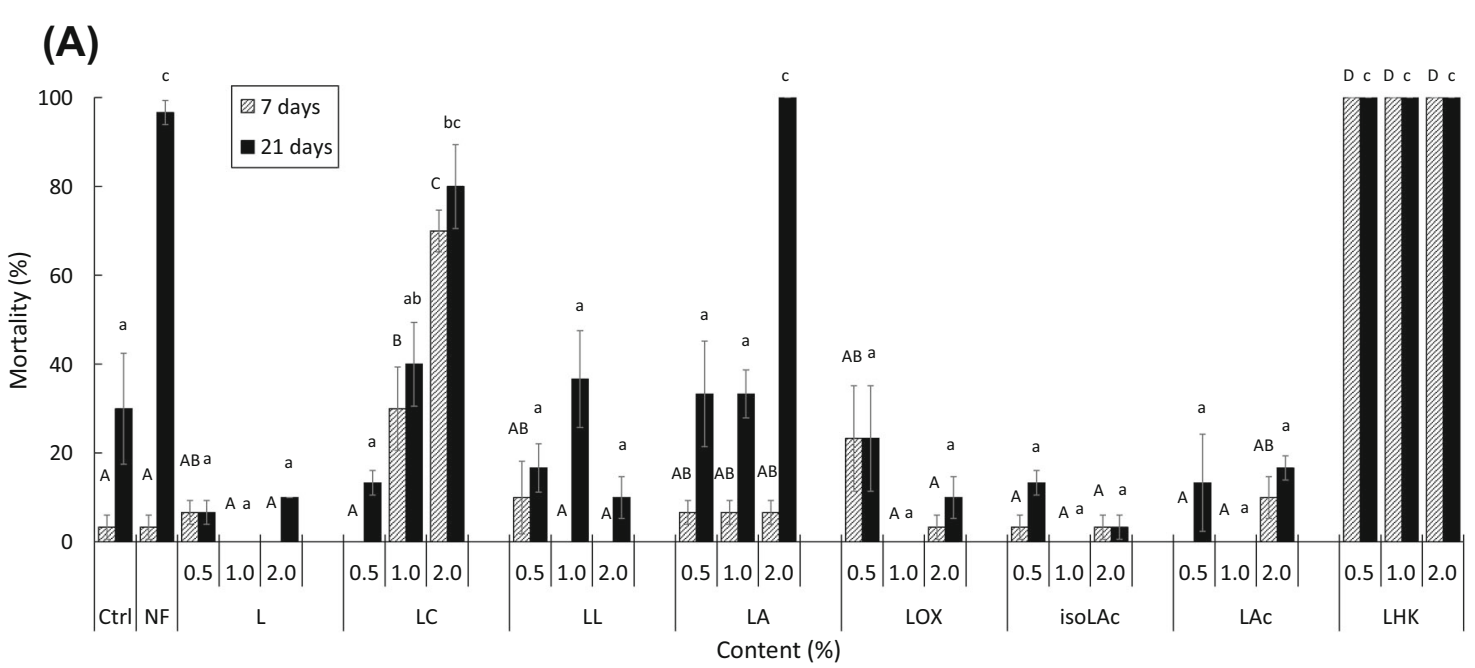

(B)

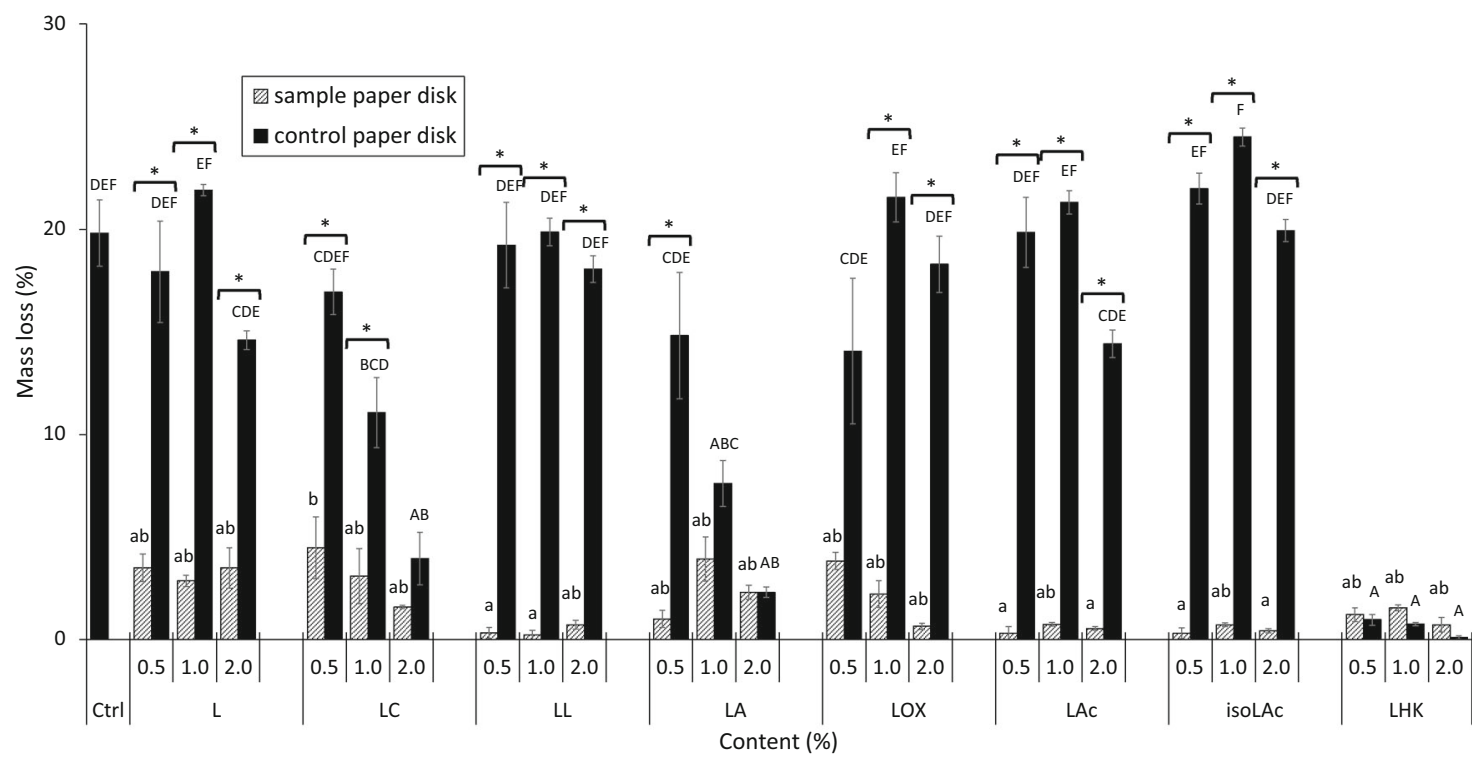

Fig. 5 a Termiticidal activities after 7 and 21 days and $\mathbf{b}$ antifeedant activities after 21 days of exposure to compounds in the dual-choice test. Ctrl, control test; $\mathbf{L}$, longifolene; LC, longicamphenylone; LL, norlongilactone; LA, longicamphenylol; LAc, longifolic acid; LOX, longifolene oxide; isoLAc, isolongifolic acid; LHK, longifolene-

condition was needed to supply energy for the formation of LHK.

\section{Mechanism of longifolene autoxidation}

The formation of autoxidation products increased gradually during the reaction at $120{ }^{\circ} \mathrm{C}$ (Table 2). To investigate the early stage of the reaction, $\mathbf{L}$ was autoxidized at $120{ }^{\circ} \mathrm{C}$ for 30 and $60 \mathrm{~min}$, and the reaction mixtures were analyzed for minor products by $\mathrm{GC}-$ MS. Longifolic acid (LOX) was detected within the first hydroxy ketone; NF, no-feed test. Bars indicate standard errors. Common letters indicate that there is no significant difference in the values. Asterisks denote a significant difference between sample and control papers; Tukey-Kramer test, $p<0.05$

30 min, and then $\mathbf{L C}$ and other products were detected at $60 \mathrm{~min}$. When pure LOX was heated at $120{ }^{\circ} \mathrm{C}$ without solvent and catalysis in the presence of air, it was converted into LC, LL, LAc, isoLAc, and LHK (Fig. 3). These results suggest that $\mathbf{L}$ was converted into various autoxidation products through the formation of LOX as an intermediate. Nayak et al. [15] reported the formation of these products following the oxidation of $\mathbf{L}$ with perbenzoic acid, and Dimitrov et al. [14] reported that the acidic products LAc and isoLAc were formed from the ozonolysis of longifolene. Both reports 
proposed a common reaction mechanism in which LOX served as an intermediate that was cleaved and subsequently oxidized. The proposed mechanism supports our experimental results. In this study, we considered that the natural autoxidation after formation of LOX proceeded in the same manner as the perbenzoic acid oxidation and the ozonolysis.

\section{Anti-termite activities of the longifolene (L) autoxidation products}

In the no-choice test, all autoxidation products isolated in this study exhibited termiticidal or antifeedant activity, although $\mathbf{L}$ did not have these activities, compared with the control test (Fig. 4). LHK had the highest termiticidal activity in this test, and most of the termites were killed in the early stage at a low concentration $(0.5 \%)$. Thus, LHK is highly toxic to termites. The termiticidal activities of the other products were concentration-dependent. Because the mass loss values of the paper disk were small in the tests of $\mathbf{L L}, \mathbf{L A c}$, and isoLAc, depending on their concentration, their termiticidal activities mainly resulted from their antifeedant activities. LC, LA, and LOX exhibited higher termiticidal activities in the no-choice test than in the nofeed test for 7 days. When testing high concentrations of LA and LOX, almost all the termites died within 21 days, and the mass loss values of the paper disk in the test were small. LA and LOX were toxic to termites, and they exhibited strong antifeedant activities. LC resulted in termite mortality in the early stage (day 7), but the mortality rate did not increase significantly until the end of the test period. Although the mass loss values of the paper disks decreased depending on the LC concentration, the values were larger than those of the other autoxidation products. Thus, LC exhibited toxicity and weak antifeedant activity for termites.

The dual-choice test was performed to investigate the termiticidal and repellent activities of these compounds. Figure 5 shows the results of the dual-choice test for $\mathbf{L}$ and its autoxidation products. The repellent activities of the products differed significantly compared with the control paper disk in the same Petri dish, as determined by a $t$ test. LC, LA, and LHK clearly exhibited termiticidal activity. LHK had strong lethal activity in both the dual-choice and no-choice tests. L, LC, LL, LOX, LA, LAc and isoLAc also exhibited repellent activity. The only anti-termite activity of $\mathbf{L}$ was its repellent activity. The above results show that the autoxidation products of $\mathbf{L}$ had various antitermite activities, such as antifeedant and/or termiticidal activities, as well as repellent activity. This indicates that L can be converted easily into more bioactive compounds by autoxidation.

\section{Conclusions}

The products of longifolene autoxidation at $120{ }^{\circ} \mathrm{C}$ were longicamphenylone, norlongilactone, longifolene oxide, longifolic acid, isolongifolic acid, and longifolene-hydroxy ketone. Longifolene was converted into the autoxidation products via the formation of longifolene oxide as an intermediate. All the autoxidation products had stronger lethal and/or antifeedant activities for termites than longifolene, and longifolene-hydroxy ketone showed potent termiticidal activity. Thus, longifolene can be converted easily into bioactive components by autoxidation.

Acknowledgements We thank Dr. Shizuo Nagahama, Professor Emeritus of Sojo University, for supplying authentic samples. This research was supported by JSPS KAKENHI (Grant No. JP15K07503).

\section{References}

1. Torssell K (1997) Natural product chemistry. Swedish Pharmaceutical Press, Stockholm, pp 42-79

2. Ashitani T, Nagahama S (1999) Direct episulfidation of caryophyllene and humulene. Nat Prod Lett 13:163-167

3. Ashitani T, Borg-Karlson AK, Fujita K, Nagahama S (2008) Reaction mechanism of direct episulfidation of caryophyllene and humulene. Nat Prod Res 22:495-498

4. Ashitani T, Kusumoto N, Borg-Karlson AK, Fujita K, Takahashi K (2013) Antitermite activity of $\beta$-caryophyllene epoxide and episulfide. Zeit Naturforsch 68C:302-306

5. Ashitani T, Garboui SS, Schubert F, Vongsombath C, Liblikas I, Pålsson K, Borg-Karlson AK (2015) Activity studies of sesquiterpene oxides and sulfides from the plant Hyptis suaveolens (Lamiaceae) and its repellency on Ixodes ricinus (Acari: Ixodidae). Exp Appl Acarol 67:595-606

6. Nagahama S, Tazaki M (1987) Terpenoides X. natural autoxidation of thujopsene. Bull Chem Soc Jpn 60:4453-4454

7. Ashitani T (2013) Bioactivity of tree extracts-autoxidation and antitermitic activity of unutilized sesquiterpene (in Japanese). Green Spirits 8:3-7

8. Ashitani T (2016) Anti-fungal and anti-insect properties of terpenoids (in Japanese). In: Ohira T, Miyazawa M (eds) Advanced technologies for terpenoids. CMC publishing, Tokyo, pp 74-81

9. Hirao N (1937) Pinene source in Japan (III) (in Japanese). Nippon Kagaku Kaishi 58:229-233

10. Nishida K, Uota $H$ (1937) Ueber den unterschied des gehalts an $\alpha$, und $\beta$-pinen im terpentinöl durch verschiedenheit des produktionsorts (in Japanese). J Jpn For Soc 19:298-306

11. Tsuruta K, Yoshida Y, Kusumoto N, Sekine N, Ashitani T, Takahashi K (2011) Inhibition activity of essential oils obtained from Japanese trees against Skeletonema costatum. J Wood Sci 57:520-525

12. Yatagai M (2016) What are terpenoids? (in Japanese) In: Ohira T, Miyazawa M (eds) Advanced technologies for terpenoids. CMC Publishing, Tokyo, pp 1-10

13. Kasano M, Matsubara Y (1978) Oxidation of longifolene with lead tetraacetate (in Japanese). J Oleo Sci 27:309-311

14. Dimitrov V, Rentsch GH, Linden A, Hesse M (2003) The ozonolysis of longifolene: a tool for the preparation of useful chiral compounds. Configuration determination of new 
stereogenic centers by NMR spectroscopy and X-ray crystallography. Helv Chem 86:106-121

15. Nayak UR, Dev S (1963) Studies in sesquiterpenes-XIX: action of perbenzoic acid on longifolene. Tetrahedron 19:2269-2280

16. Nayak UR, Dalavoy VS, Deodhar VB (1989) Benzyltriethylammonium chloride-catalyzed permanganate oxidation of longifolene. Ind J Chem 28B:1055-1056

17. Bowman RM, Chambers A, Jackson WR (1966) The epoxidation of p-menth-1-and-3-ene. J Chem Soc C 612-615. doi:10.1039/ J39660000612

18. Nomura M, Fujihara Y (1988) Decomposition reaction of longifolene oxide, isolongifolene oxide and $\beta$-caryophyllene oxide with acids in the presence of synthetic zeolites. J Oleo Sci 37:97-101

19. Kusumoto N, Ashitani T, Hayasaka Y, Murayama T, Ogiyama K, Takahashi K (2009) Antitermitic activities of abietane-type diterpenes from Taxodium distichum cones. J Chem Ecol 35:635-642

20. Morikawa T, Ashitani T, Kofujita H, Takahashi K (2014) Antitermitic activity of extracts from Chamaecyparis obtusa branch heartwood. Eur J Wood Wood Prod 72:651-657

21. Lhomme J, Ourisson G (1964) Longifolene (VIII)—mechanism of oxidation of longifolene by peracids (in French). Bull Soc Chem Fr 5:1888-1889

22. Joshi AP, Nayak UR, Dev S (1976) Studies in sesquiterpenes-L 3hydroxylongifolaldehyde, the elusive intermediate in the abnormal perbenzoic acid oxidation of longifolene. Tetrahedron 32:1423-1425

23. Tanaka J, Takabe K, Kawakita M, Ito M (1978) Katagiri T (1978), Abnormal ozonolysis of methylenebicyclo [m. n. 1] alkanes (in Japanese). Nippon Kagaku Kaishi 2:284-287. doi:10. 1246/nikkashi.1978.284 